\title{
Tongue Color Analysis for Medical Application
}

\author{
Bob Zhang, ${ }^{1,2}$ Xingzheng Wang, ${ }^{2}$ Jane You, $^{2}$ and David Zhang ${ }^{2}$ \\ ${ }^{1}$ Department of Electrical \& Computer Engineering, Carnegie Mellon University, Pittsburgh, PA 15213, USA \\ ${ }^{2}$ Biometrics Research Center, Department of Computing, The Hong Kong Polytechnic University, Hong Kong
}

Correspondence should be addressed to Bob Zhang; yibo1@ece.cmu.edu

Received 6 December 2012; Accepted 3 March 2013

Academic Editor: Jong Yeol Kim

Copyright (C) 2013 Bob Zhang et al. This is an open access article distributed under the Creative Commons Attribution License, which permits unrestricted use, distribution, and reproduction in any medium, provided the original work is properly cited.

\begin{abstract}
An in-depth systematic tongue color analysis system for medical applications is proposed. Using the tongue color gamut, tongue foreground pixels are first extracted and assigned to one of 12 colors representing this gamut. The ratio of each color for the entire image is calculated and forms a tongue color feature vector. Experimenting on a large dataset consisting of 143 Healthy and 902 Disease (13 groups of more than 10 samples and one miscellaneous group), a given tongue sample can be classified into one of these two classes with an average accuracy of $91.99 \%$. Further testing showed that Disease samples can be split into three clusters, and within each cluster most if not all the illnesses are distinguished from one another. In total 11 illnesses have a classification rate greater than $70 \%$. This demonstrates a relationship between the state of the human body and its tongue color.
\end{abstract}

\section{Introduction}

The human tongue contains numerous features that can be used to diagnose disease, with color features being the most important [1-4]. Traditionally, medical practitioners would examine these color features based on years of experience [511]. However, ambiguity and subjectivity are always accompanied with their diagnostic result. To remove these qualitative aspects, tongue color analysis can be objectively analyzed through its color features, which offers a new way to diagnose disease, one that minimizes the physical harm inflicted to patients (compared with other medical examinations).

A literature review on this topic revealed only a few papers where color features from the tongue are the main component used to diagnose disease. Reference [12] used tongue color along with qualitative and quantitative analysis to examine 207 patients suffering from lung cancer. The patients were split into four syndrome groups according to Chinese Medicine, and the CIELAB color model was used for quantitative classification. They reported significant statistical difference between the four groups when it came to each tongue's CIELAB value. The correct classification rate of each group was $69.4 \%, 54.4 \%, 72.2 \%$, and $54.4 \%$, respectively. A color metrics was utilized in [13] to diagnose appendicitis using tongue images. 798 tongue samples were captured from 399 patients (two samples from each person), consisting of common illnesses such as diabetes, pancreatitis, and hypertension, along with 114 images from tongues affected by appendicitis. The samples were captured using a specially designed device consisting of a 3-CCD digital camera, two D65 lights and calibrated with a white color plate. Four color spaces (RGB, CIExyY, CIELUV, and CIELAB) were evaluated to find the best combination. In their work they reported a correct classification of appendicitis to be $66.67 \%$. Pancreatitis (29 samples) and appendicitis (53 samples) tongue images again appeared in [14], albeit this time with 56 normal samples. These images were captured with a device designed by their research center consisting of a lens, CCD sensor board, two D65 lights, and video frame grabber. Assessing the same four spaces as [13], the experimental results showed that normal and pancreatitis as well as appendicitis and pancreatitis can be linearly separated using color features.

In [12] the dataset was quite small and only one disease was analyzed. The patients were also diagnosed using Chinese Medicine. As for [13] its dataset was substantially larger but only appendicitis was classified. The samples in [14] include two illnesses as well as normal, but their sizes are too small to have any statistical significance. Both works in 


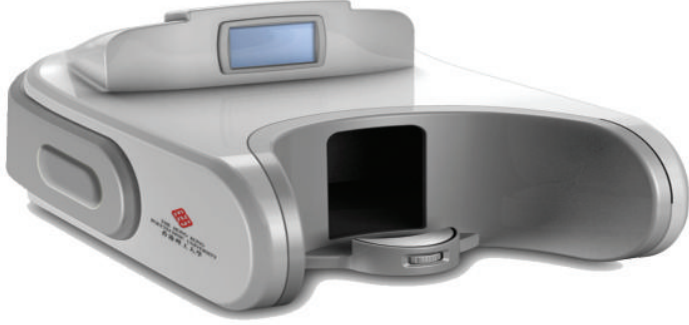

FIGURE 1: Tongue capture device.

TABLE 1: Disease class statistics listing the ID, name, and number of samples.

\begin{tabular}{llc}
\hline $\begin{array}{l}\text { Disease } \\
\text { ID }\end{array}$ & Disease name & $\begin{array}{c}\text { No. of } \\
\text { samples }\end{array}$ \\
\hline 1 & Chronic Kidney Disease & 141 \\
2 & Diabetes & 69 \\
3 & Nephritis & 10 \\
4 & Hypertension & 66 \\
5 & Verrucous Gastritis & 25 \\
6 & Pneumonia & 10 \\
7 & Nephritic Syndrome & 10 \\
8 & Chronic Cerebral Circulation Insufficiency & 14 \\
9 & Upper Respiratory Tract Infection & 12 \\
10 & Erosive Gastritis & 10 \\
11 & Coronary Heart Disease & 13 \\
12 & Chronic Bronchitis & 11 \\
13 & Mixed Hemorrhoid & 11 \\
14 & Miscellaneous & 500 \\
\hline
\end{tabular}

$[12,14]$ did not use any image correction to ensure uniform feature extraction and analysis under different operating conditions. Also, [12-14] used some variation of the CIE color space to embody the tongue colors, which may not be very accurate. Therefore, there is a lack of any work on an indepth systematic tongue color analysis system for medical applications, one that accurately captures the images and represents its colors using a tongue color gamut [15]. In this paper such an application is described to address these problems. Tongue images are first captured using a specifically designed device with image correction. Afterwards, the images are segmented [16] with the background removed and tongue foreground remaining. Color features from each pixel are then extracted and assigned to 1 of 12 colors symbolizing the tongue color gamut [15]. This produces the tongue color feature vector. Experimental results were carried out on a large scale dataset consisting of 143 Healthy and 902 Disease samples (diagnosed using Western Medicine) taken from Guangdong Provincial Hospital of Traditional Chinese Medicine, Guangdong, China. The Disease class was composed of 13 specific illnesses (with at least 10 samples in each group) and one sizeable miscellaneous group (made up of various illnesses). Classification was performed between the illnesses in addition to Healthy versus Disease.

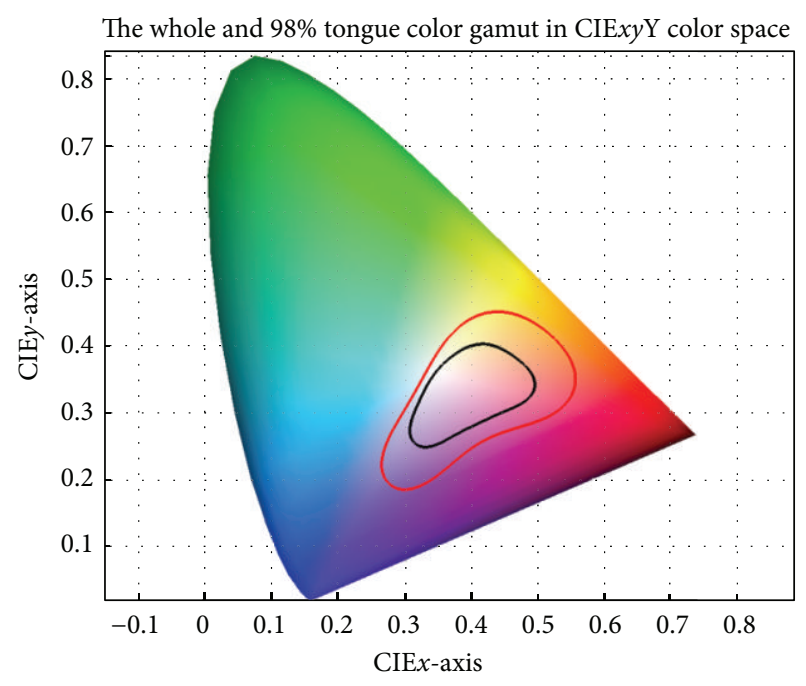

FIgURE 2: A color gamut in the CIExyY color space depicting the tongue color gamut inside the red boundary. Furthermore, 98\% of the tongue color gamut can be located within the black boundary.

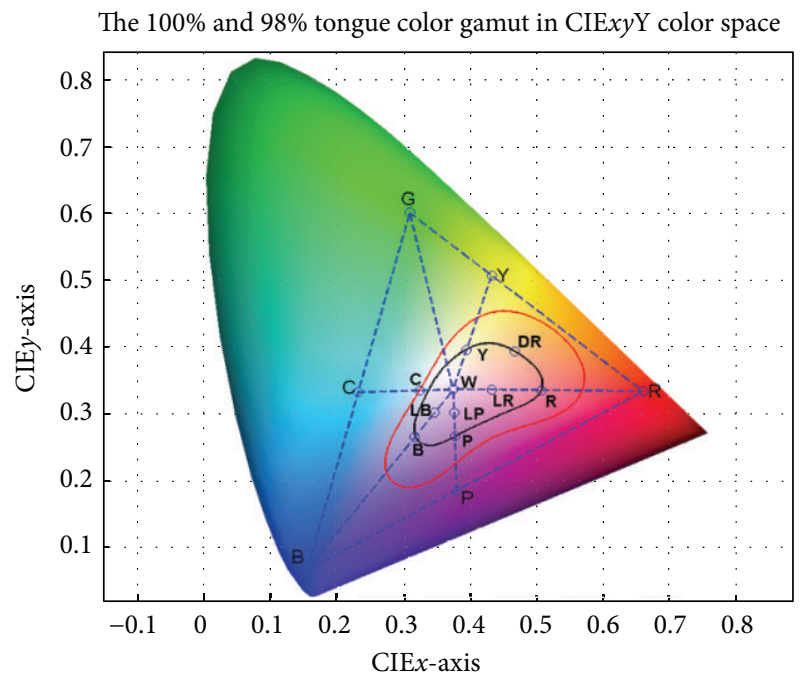

FIGURE 3: The tongue color gamut can be represented using several points by drawing lines from the RGB color space.

The rest of this paper is organized as follows. An introduction to the tongue image acquisition device and dataset used is given in Section 2. Section 3 summarizes the tongue color gamut and explains how color features are extracted using it. In Section 4 classification between the two classes of Healthy and Disease is performed. Following this, illnesses in the Disease class are classified. Finally, concluding remarks are made in Section 5.

\section{Materials}

The tongue database is composed of 1045 images (one image per person) split into 143 Healthy and 902 Disease captured at 


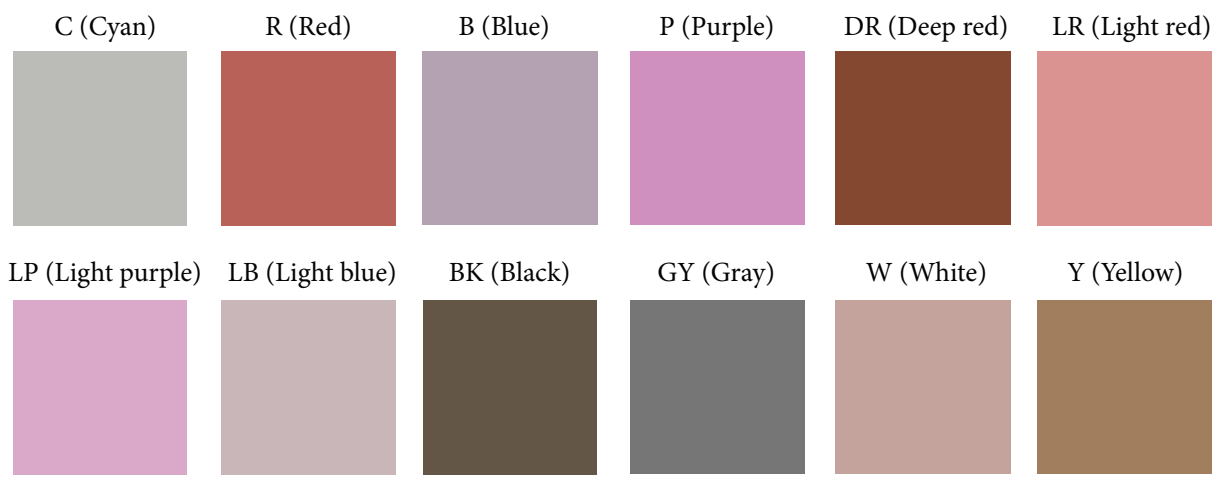

FIGURE 4: 12 colors representing the tongue color gamut with its label on top.

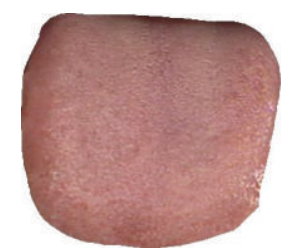

$v=\left(\begin{array}{ll}0.001 & 17.067006 .00454 .7220 .0290 .2055 .5514 .7663 .7117 .899\end{array}\right)$

C- $0.001 \%$

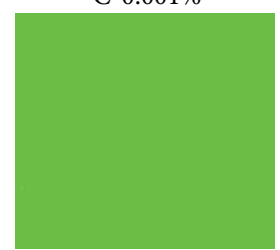

DR-6.004\%

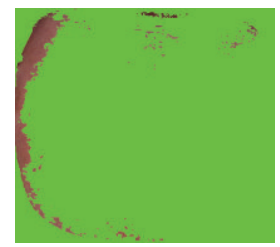

BK-5.551\%

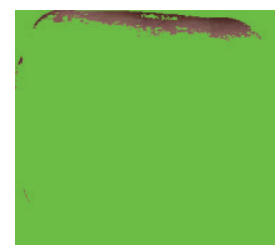

R-17.067\%

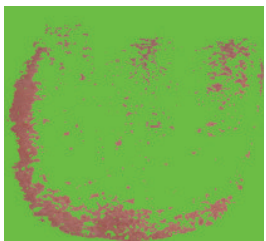

LR- $54.722 \%$

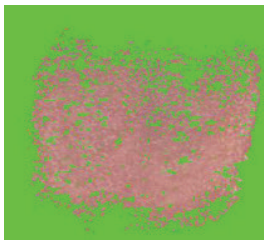

GY- $4.766 \%$

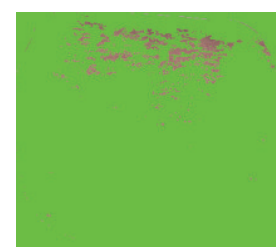

B- $0 \%$

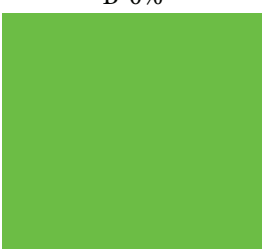

LP- $0.029 \%$

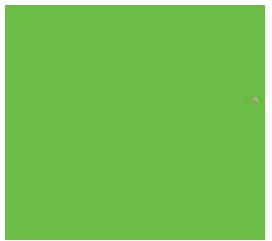

W-3.711\%

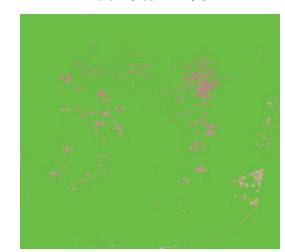

P- $-0 \%$

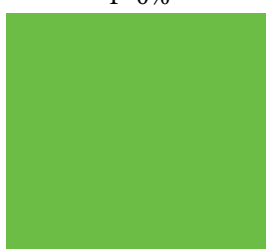

LB- $0.205 \%$

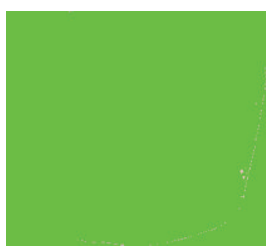

Y-7.899\%

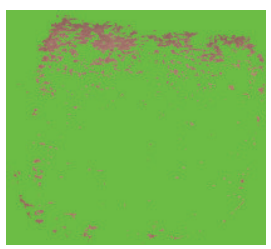

FIGURE 5: Healthy tongue sample, its tongue color feature vector and corresponding 12-color makeup with most of the pixels classified as LR. 


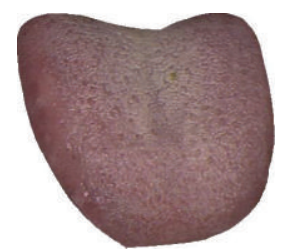

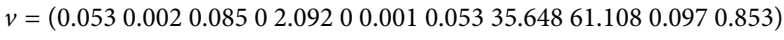

C- $0.053 \%$

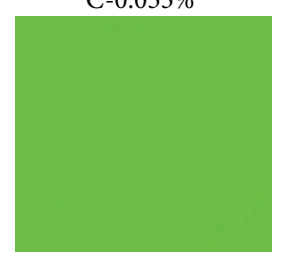

DR-2.092\%

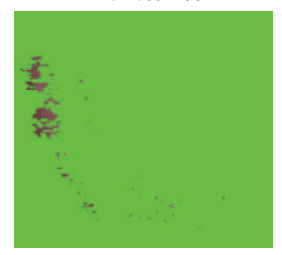

BK-35.648\%

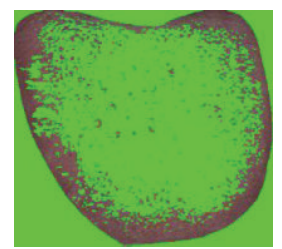

R- $0.002 \%$

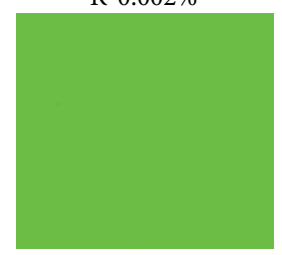

LR-0\%

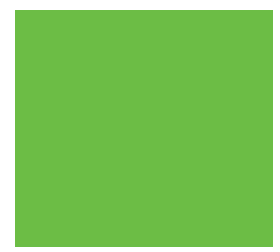

GY-61.108\%

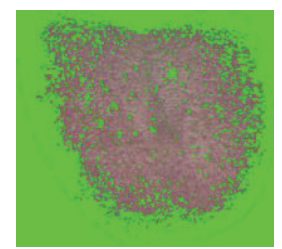

B- $0.085 \%$

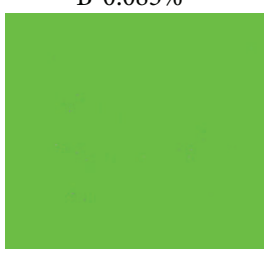

LP- $0.001 \%$

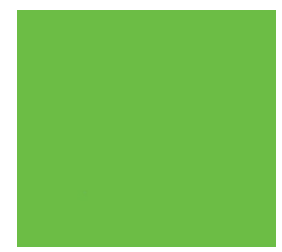

W- $0.097 \%$

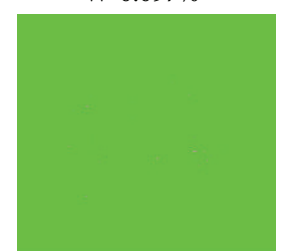

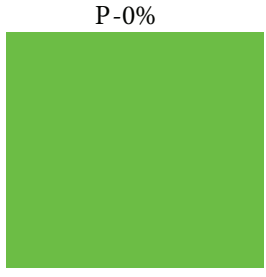

LB- $0.053 \%$

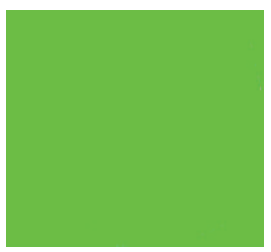

Y- $0.853 \%$

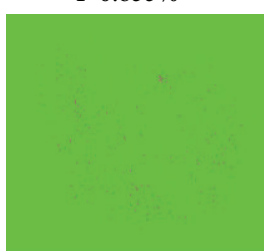

FIGURE 6: Disease tongue sample, its tongue color feature vector and corresponding 12-color makeup with most of the pixels classified as GY.

Guangdong Provincial Hospital of Traditional Chinese Medicine, Guangdong, China. The patients' consent was obtained according to the Declaration of Helsinki and the Ethical Committee of the Institution in which the work was performed approved it. The capture device used was a three-chip CCD camera with 8 bit resolution and two D65 fluorescent tubes placed symmetrically around the camera in order to produce a uniform illumination. The images captured were color corrected [17] to eliminate any noise caused by variations of illumination and device dependency. This allows consistent feature extraction and classification in the following steps. Figure 1 shows the capture device. Healthy samples were verified through a blood test and other experiments. If indicators from the tests fall within a certain range they were deemed fit. In the Disease class, samples were collected from inpatients with illnesses determined by their admission note, diagnosed using Western Medical practices. Inpatients suffering from the same illness were grouped together into a single class. In total there were 13 ailment groups (with at least 10 samples) and one miscellaneous group containing various illnesses. A summary of the Disease class breakdown is given in Table 1. Please note any future reference to a specific illness in Table 1 will be made using its Disease ID.

\section{Methods}

The following section describes how color features are extracted from tongue images. The tongue color gamut is first summarized in Section 3.1. In Section 3.2, every foreground tongue pixel is compared to 12 colors representing the tongue color gamut and assigned its nearest color. This forms the color features.

3.1. Tongue Color Gamut. The tongue color gamut [15] represents all possible colors that appear on the tongue surface and exists within the red boundary shown in Figure 2 (CIE$x y$ chromaticity diagram). Further investigation revealed that $98 \%$ of the points lie inside the black boundary. To represent the tongue color gamut using 12 colors, the RGB color space is employed and plotted in Figure 3. On the RG line a point $Y$ (Yellow) is marked. Between $\mathrm{RB}$ a point $\mathrm{P}$ (Purple) is marked and $\mathrm{C}$ (Cyan) is marked between GB. The center of the RGB color space is calculated and designated as W (White), the first of the 12 colors (see Figure 3). Then, for each R (Red), B (Blue), Y, P, and C point, a straight line is drawn to W. Each time these lines intersect the tongue color gamut, a new color is added to represent the 12 colors. This accounts for R, Y, C, B, 

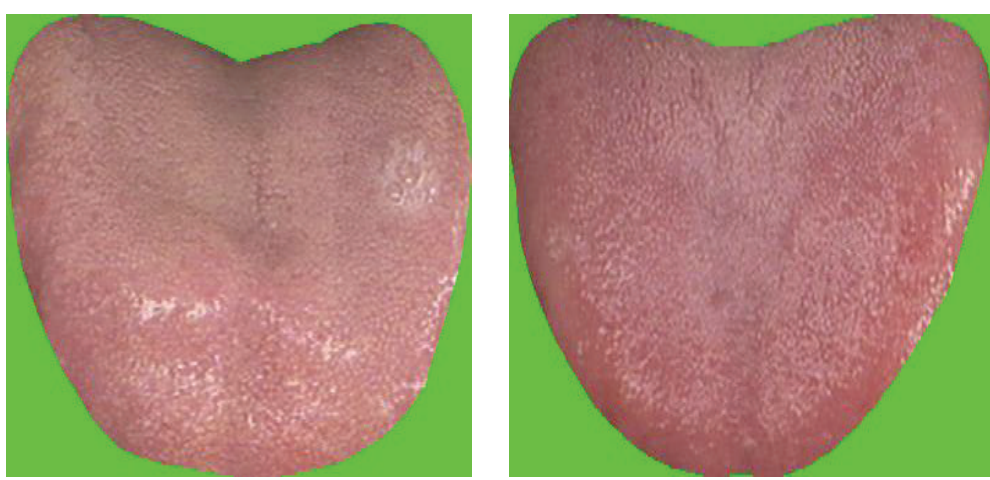

Healthy

(a)
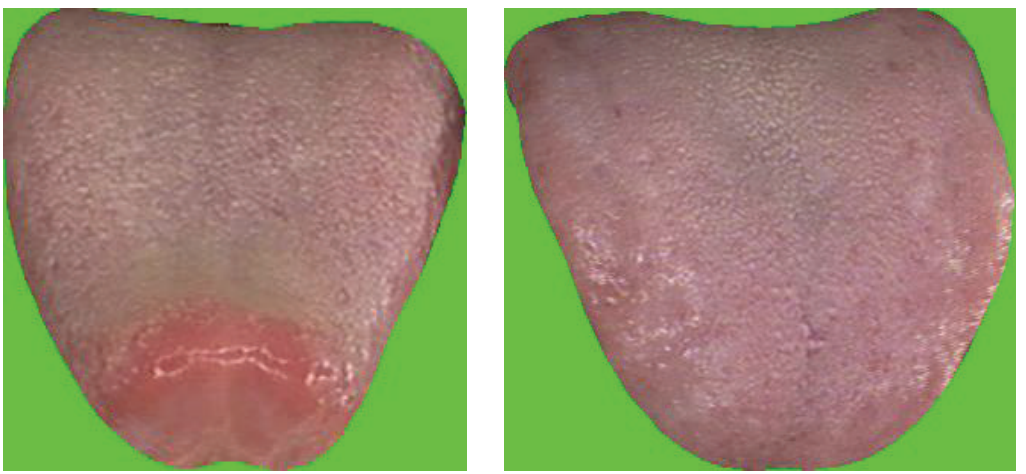

Disease

(b)
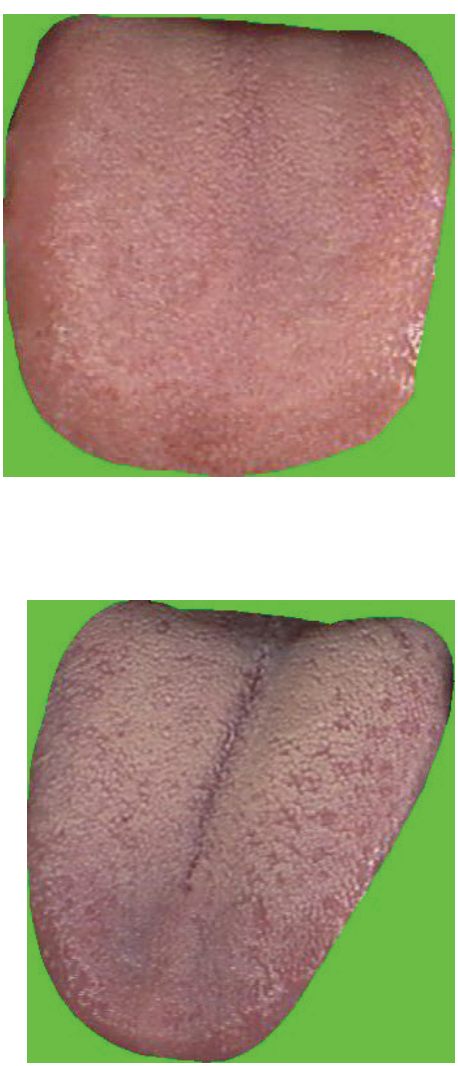

(n)

Figure 7: Three typical Healthy (a) and Disease (b) samples.

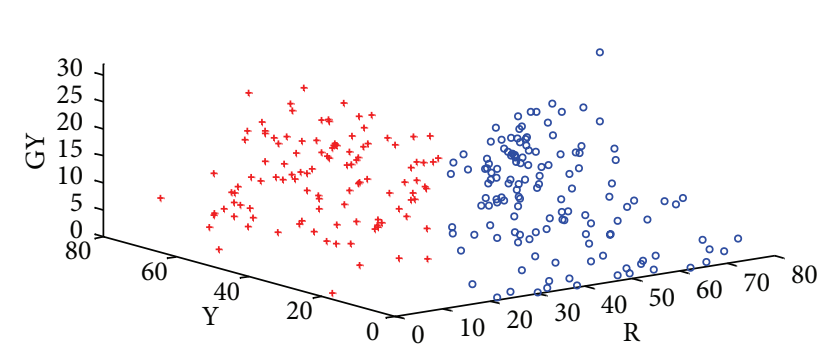

+ Cluster 1

- Cluster 2

Figure 8: Plot of Cluster 1 versus Cluster 2.

and P. LR (Light red), LP (Light purple), and LB (Light blue) are midpoints between lines from the black boundary to $\mathrm{W}$, while DR (Deep red) is selected as no previous point occupies that area. More details about the tongue color gamut can be found in [15]. GY (Gray) and BK (Black) are not shown in Figure 3 since both belong to grayscale.

The 12 colors representing the tongue color gamut are extracted from Figure 3 and shown in Figure 4 as a color square with its label on top. Correspondingly, its RGB and CIELAB values are given in Table 2.

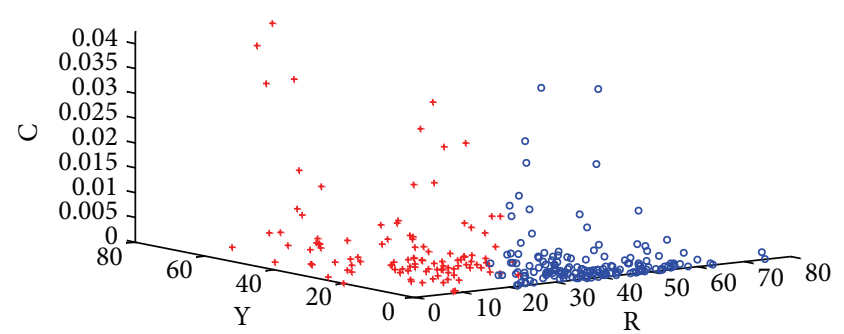

+ Cluster 1
- Cluster 3

Figure 9: Plot of Cluster 1 versus Cluster 3.

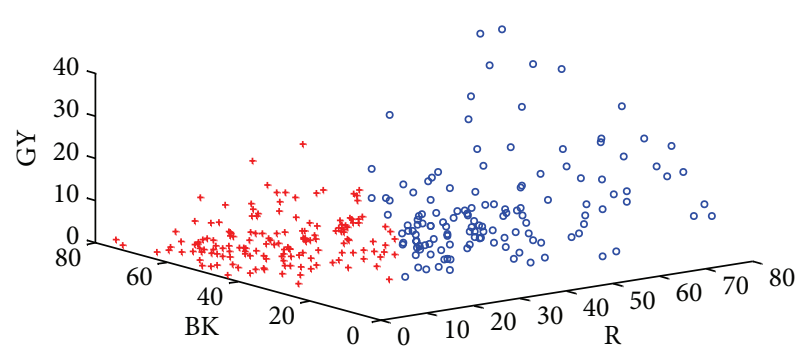

+ Cluster 3

- Cluster 2

Figure 10: Plot of Cluster 3 versus Cluster 2. 

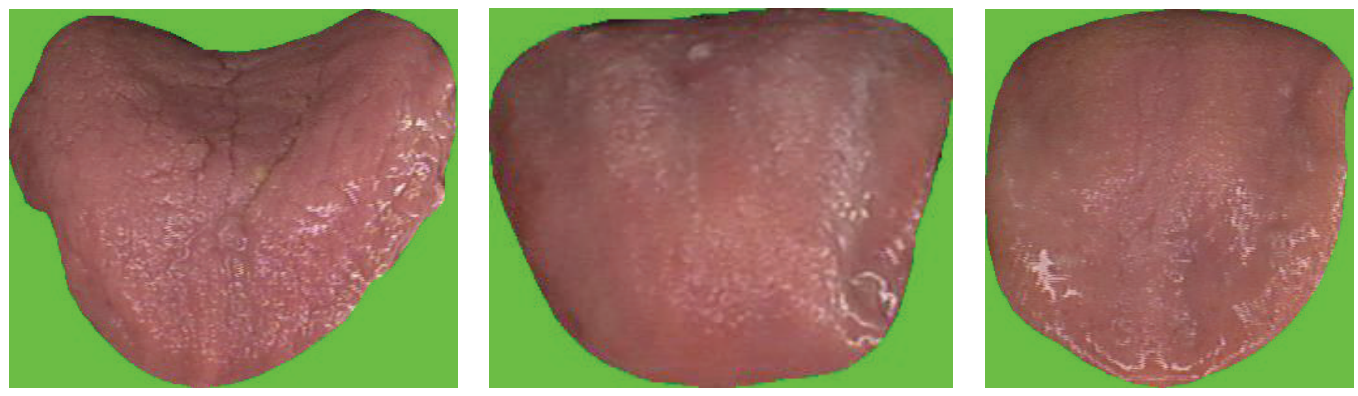

Cluster 1

(a)
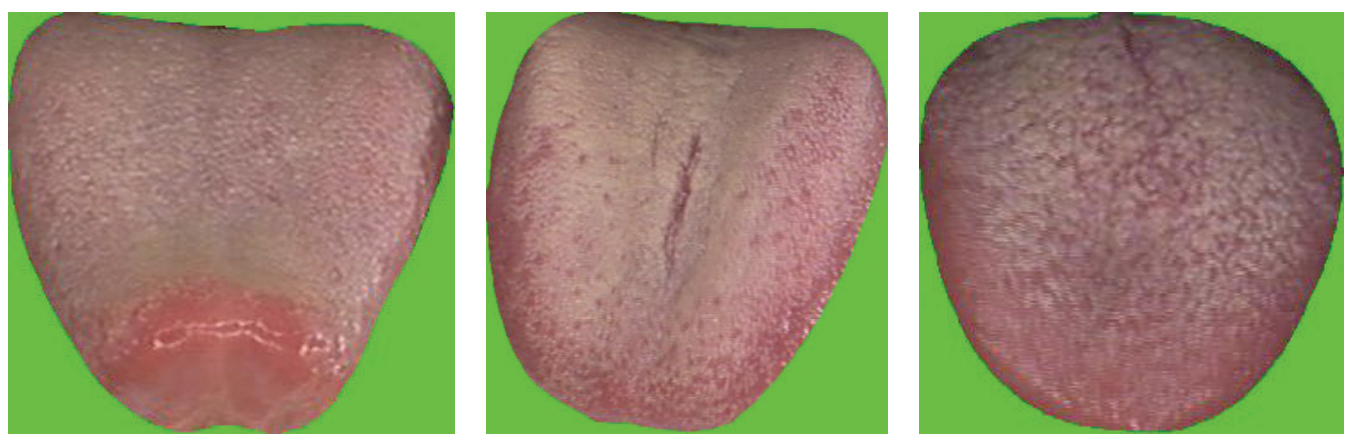

Cluster 2

(b)
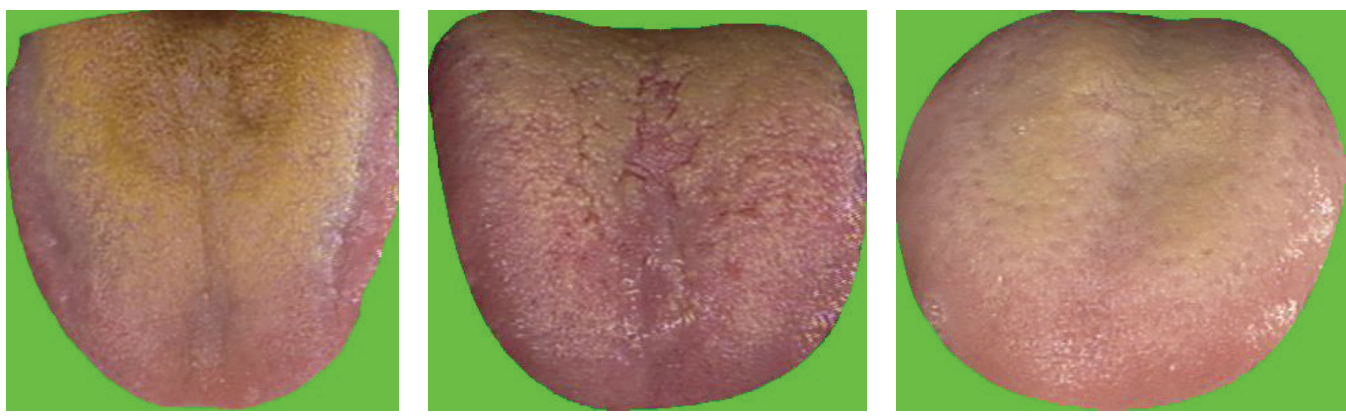

Cluster 3

(c)

FIgURE 11: Three typical samples from each cluster.
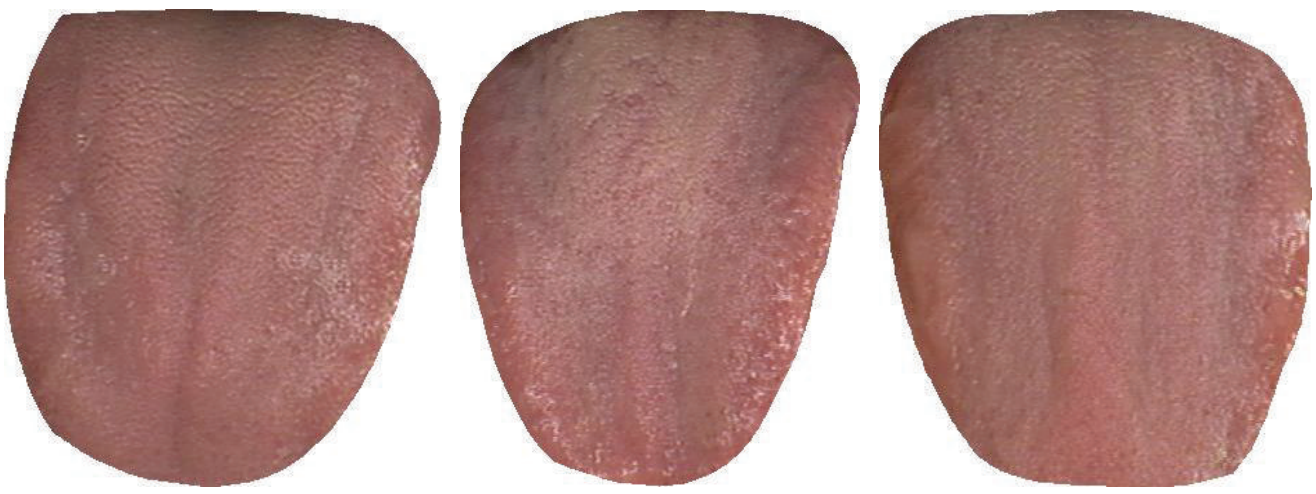

FIGURE 12: Three typical samples from Disease 1. 


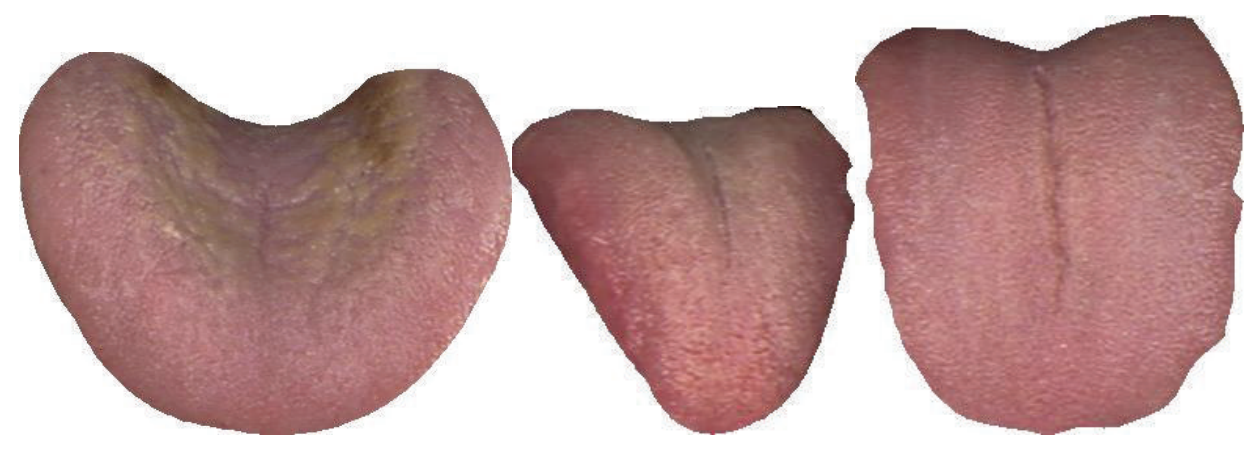

FIgURE 13: Three typical samples from Disease 3.
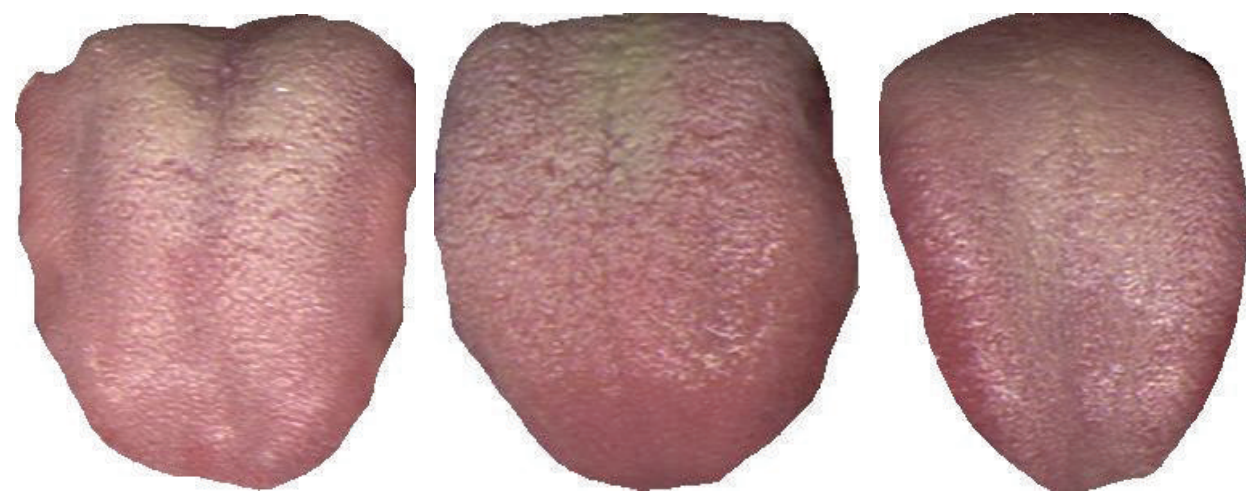

Figure 14: Three typical samples from Disease 5.
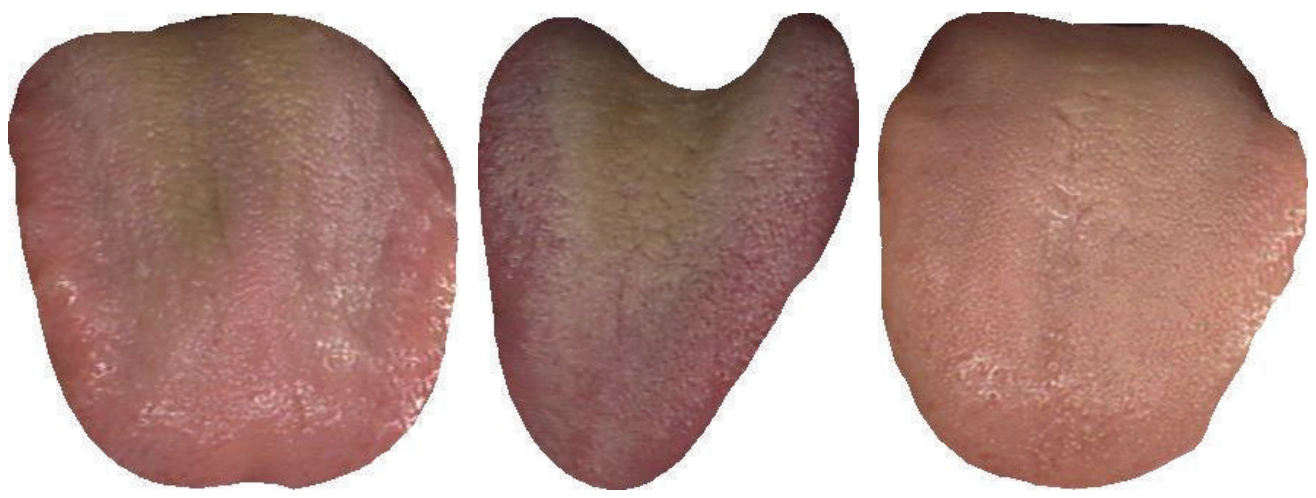

FIgURE 15: Three typical samples from Disease 6.
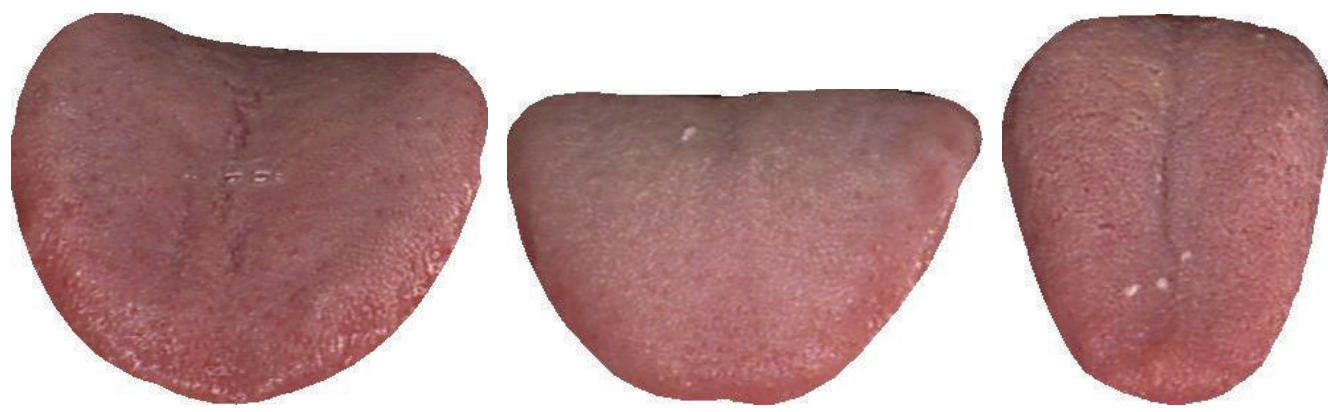

Figure 16: Three typical samples from Disease 7. 

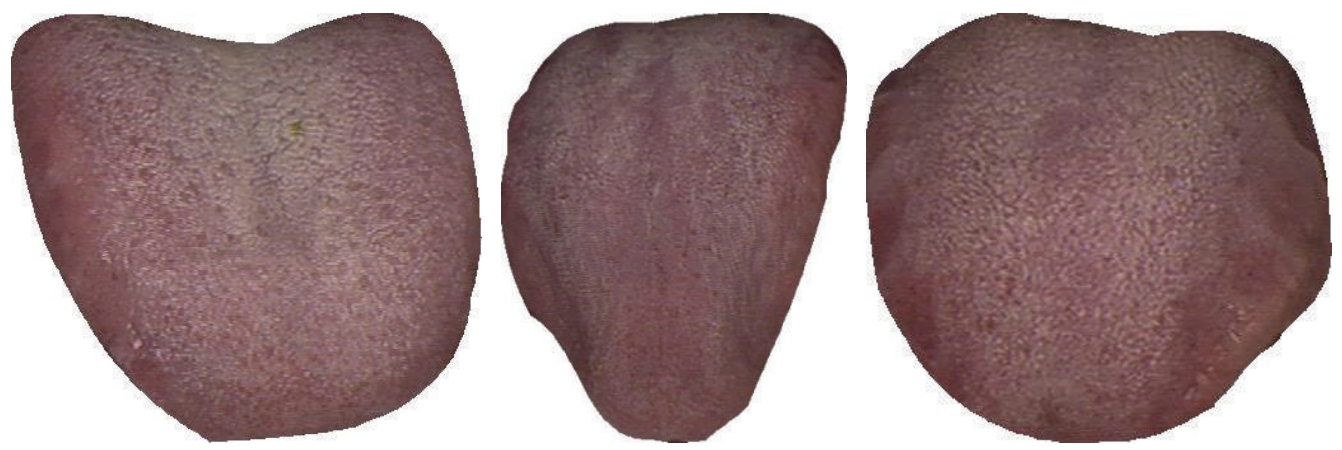

Figure 17: Three typical samples from Disease 8.
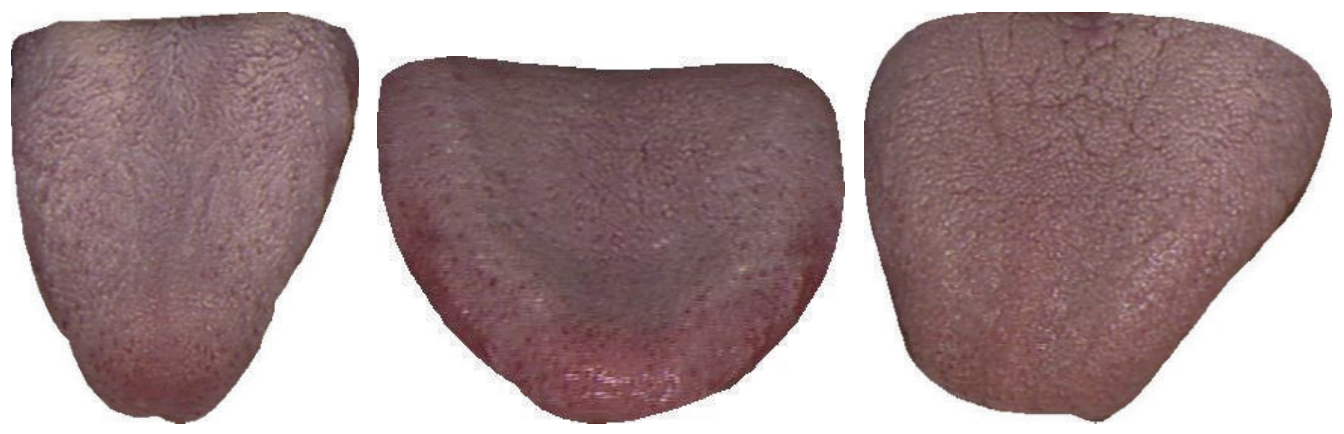

FIgURE 18: Three typical samples from Disease 9.
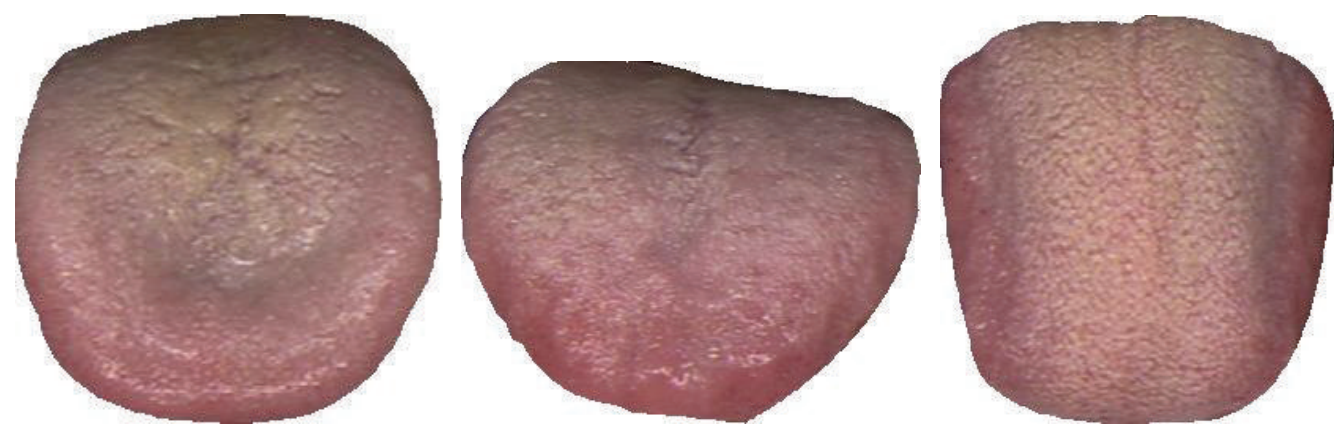

Figure 19: Three typical samples from Disease 10.
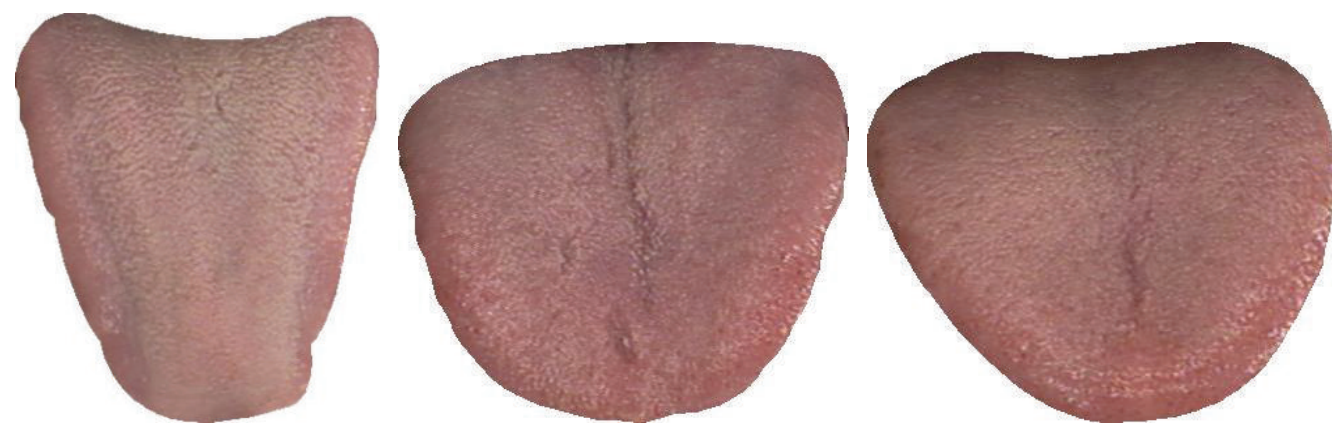

Figure 20: Three typical samples from Disease 11. 

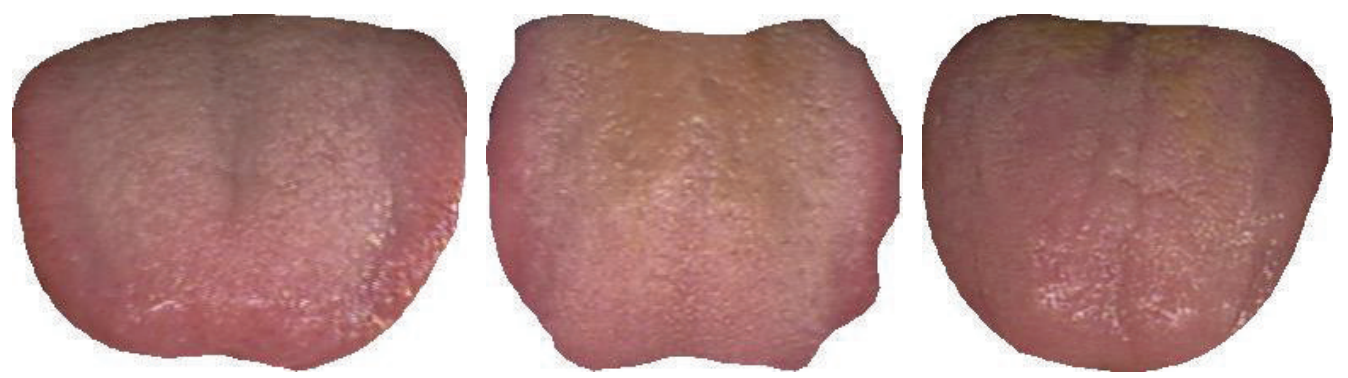

FIGURE 21: Three typical samples from Disease 12.
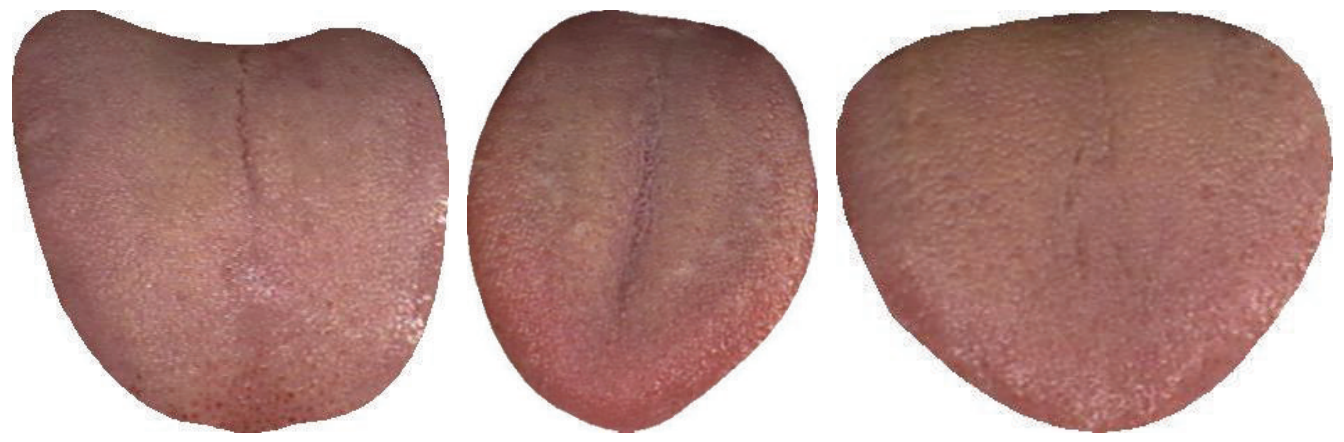

FIgURE 22: Three typical samples from Disease 13.

3.2. Tongue Color Features. Given a tongue image, segmentation is first applied to locate all foreground tongue pixels [16]. Having located each pixel its corresponding RGB value is extracted and converted to CIELAB [18] by first converting RBG to CIEXYZ using

$$
\left[\begin{array}{l}
X \\
Y \\
Z
\end{array}\right]=\left[\begin{array}{lll}
0.4124 & 0.3576 & 0.1805 \\
0.2126 & 0.7152 & 0.0722 \\
0.0193 & 0.1192 & 0.9505
\end{array}\right]\left[\begin{array}{l}
\mathrm{R} \\
\mathrm{G} \\
\mathrm{B}
\end{array}\right]
$$

followed by CIEXYZ to CIELAB via

$$
\begin{gathered}
L^{*}=116 f\left(\frac{Y}{Y_{0}}\right)-16, \\
a^{*}=500\left[f\left(\frac{X}{X_{0}}\right)-f\left(\frac{Y}{Y_{0}}\right)\right], \\
b^{*}=200\left[f\left(\frac{Y}{Y_{0}}\right)-f\left(\frac{Z}{Z_{0}}\right)\right],
\end{gathered}
$$

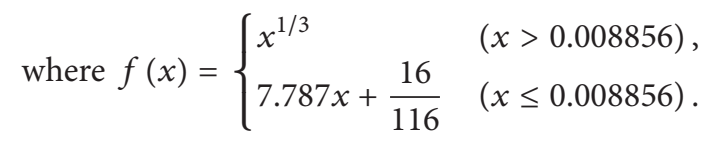

In (2), $X_{0}, Y_{0}$, and $Z_{0}$ are the CIEXYZ tristimulus values of the reference white point. The $L A B$ values are then compared to 12 colors from the tongue color gamut (see Table 2) and assigned the color which is closest to it (measured using Euclidean distance). After calculating all tongue foreground pixels, the total of each color is summed

\begin{tabular}{|c|c|c|}
\hline Color & [R G B] & [L A B] \\
\hline C (Cyan) & {$\left[\begin{array}{llll}188 & 188 & 185\end{array}\right]$} & [76.0693-0.5580 1.3615] \\
\hline $\mathrm{R}(\mathrm{Red})$ & [189 9991$]$ & {$\left[\begin{array}{lllll}52.2540 & 34.8412 & 21.3002\end{array}\right]$} \\
\hline B (Blue) & {$\left[\begin{array}{lll}183 & 165 & 180\end{array}\right]$} & [69.4695 $9.5423-5.4951]$ \\
\hline P (Purple) & [226 142 214] & {$\left[\begin{array}{l}69.4695 \\
42.4732-23.8880\end{array}\right]$} \\
\hline DR (Deep red) & [136 72 49] & [37.8424 24.550325 .9396$]$ \\
\hline LR (Light red) & {$\left[\begin{array}{lll}227 & 150 & 147\end{array}\right]$} & [69.4695 28.4947 13.3940] \\
\hline LP (Light purple) & [225 173 207] & [76.0693 $24.3246-9.7749]$ \\
\hline LB (Light blue) & [204 183 186] & [76.0693 7.89170 .9885$]$ \\
\hline BK (Black) & {$\left[\begin{array}{lll}107 & 86 & 56\end{array}\right]$} & [37.8424 3.9632 20.5874] \\
\hline GY (Gray) & {$\left[\begin{array}{llll}163 & 146 & 143\end{array}\right]$} & [61.6542 5.7160 3.7317] \\
\hline W (White) & {$\left[\begin{array}{llll}200 & 167 & 160\end{array}\right]$} & [70.9763 10.98438 .2952$]$ \\
\hline Y (Yellow) & {$\left[\begin{array}{llll}16 & 129 & 93\end{array}\right]$} & [56.3164 9.5539 24.4546] \\
\hline
\end{tabular}
and divided by the number of pixels. This ratio of the 12 colors forms the tongue color feature vector $v$, where
TABLE 2: RGB and CIELAB values of the 12 colors.

$v=\left[c_{1}, c_{2}, c_{3}, c_{4}, c_{5}, c_{6}, c_{7}, c_{8}, c_{9}, c_{10}, c_{11}, c_{12}\right]$ and $c_{i}$ represents the sequence of colors in Table 2 . As an example, the color features of two tongues are shown in visual form (refer to Figures 5 and 6 ) along with its extracted tongue color feature vectors, where the original image is decomposed into one of the 12 colors. Figure 5 is from a Healthy sample and Figure 6 is from a Disease sample. In the Healthy sample the majority of pixels are LR and for Disease it is GY.

The mean colors of Healthy and Disease are displayed in Table 3 along with three typical samples from each class shown in Figure 7. Disease tongues have a higher ratio in $\mathrm{R}$, DR, BK, GY, and Y according to Table 3. On the other hand, LR and $\mathrm{W}$ are greater in Healthy. Only 7 colors are listed out of the 12 as the remaining 5 have ratios less than $1 \%$. 
TABLE 3: Mean of the color features for Healthy and Disease.

\begin{tabular}{lccccccc}
\hline & R & DR & LR & BK & GY & W & Y \\
\hline Healthy & 20.9284 & 5.6679 & 33.8483 & 8.2356 & 14.5583 & 7.9166 & 8.0485 \\
Disease & 28.2901 & 15.5951 & 11.0277 & 15.4325 & 16.2247 & 2.4990 & 10.6382 \\
\hline
\end{tabular}

\section{Results and Discussion}

In this section classification using color features is described. Classification between Healthy versus Disease is first given in Section 4.1, while illnesses in Disease are classified in Section 4.2.

4.1. Healthy versus Disease Classification. Table 4 shows the classification rate between Healthy versus Disease on the test data. Half the images were randomly selected from either class to represent the training set and the remaining samples assigned to the test set. The training data in each class are the mean tongue color features of Healthy and Disease. To reduce the number of tongue color features, feature selection with sequential forward search was implemented. Both $k$ NN [19] and SVM [19] using a quadratic kernel were tested producing the same result as can be seen in Table 4. This means for $k$-NN and SVM the tongue color feature vector of the training set consisting of Healthy and Disease was placed in an $n$-dimensional space. Each tongue color feature vector representing the test set was mapped to this space and classified depending on its classification rule ( $k$-NN or SVM).

4.2. Typical Disease Analysis. With Healthy versus Disease separated the next step is to examine whether certain illnesses within the Disease class can be distinguished from one another. All 13 illnesses were grouped into three clusters by FCM [19], with Table 5 illustrating which cluster each illness belongs to. The mean tongue color features of each cluster are shown in Table 6. R, DR, and LR are greater in Cluster 3. Cluster 2 has higher concentrations of GY, BK, and W, while $\mathrm{Y}$ is more significant in Cluster 1.

Table 7 shows the classification rate of the three clusters calculated in groups of two. In each case the two clusters in question are clearly separable as seen in this table and Figures 8,9 , and 10 . Three typical samples from each cluster are depicted in Figure 11. From a visual perspective the tongue color features in each cluster are quite different compared to the rest.

Next, each cluster is examined one by one to determine whether illnesses within it can be classified. This is accomplished by comparing illnesses inside the cluster and removing the illness with the highest classification. The process is repeated until all illnesses have been classified. The same experimental setup described in Section 4.1 was applied, where half the images are randomly selected for training and test sets. Both $k$-NN and SVM were used as the classifiers along with sequential forward search for feature selection. An illness is considered successfully classified if its average accuracy is greater than or equal to $70 \%$. The average accuracies stated in the following paragraph represents only SVM. For a complete list of the results please refer to Table 8 .
TABLE 4: Classification result between Healthy versus Disease using $k$-NN and SVM.

\begin{tabular}{lc}
\hline Classification method & Average accuracy \\
\hline$k$-NN & $91.99 \%$ \\
SVM & $91.99 \%$ \\
\hline
\end{tabular}

TABLE 5: Distribution of the illnesses within the clusters.

\begin{tabular}{lc}
\hline Cluster number & Disease group \\
\hline 1 & 113 \\
2 & 57891012 \\
3 & 234611 \\
\hline
\end{tabular}

Diseases 1 and 13 in Cluster 1 are separable with an average accuracy of $76.08 \%$. In Cluster 2, Disease 7 can be first removed as its classification rate of $93.06 \%$ is the highest amongst the six illnesses. Diseases 10, 8, and 9 are subsequently taken out in that order which leaves illnesses 5 and 12 (classification rate of $81.45 \%$ ). Looking at Cluster 3 , Disease 6 with a classification rate of $74.05 \%$ is initially removed from the pack. This is followed by Diseases 3 and 11 leaving 2 and 4 which produced the lowest classification result of $54.41 \%$. Table 8 summarizes this result. Diseases 1, 3, 5, 6, 7, $8,9,10,11,12$, and 13 achieved an average accuracy greater than $70 \%$ and therefore deemed successfully classified. Typical samples of the successfully classified illnesses are shown in Figures 12, 13, 14, 15, 16, 17, 18, 19, 20, 21, and 22.

As part of the future work we plan on returning to Guangdong Provincial Hospital of Traditional Chinese Medicine and collect more diseased tongue images. Color features (discussed in Section 3.2) will be extracted from these new images before combining it with the previous batch. The experimental results in the form of Healthy versus Disease classification and typical disease analysis will be recalculated in order to further validate its statistical accuracy.

\section{Conclusion}

Given a tongue image the tongue color analysis system is able to first distinguish Healthy versus Disease with an average accuracy of $91.99 \%$. If the image is from Disease it is further assigned to one of three clusters. From these clusters 11 illnesses can be successfully classified given a classification rate of at least $70 \%$. The proposed method uses a special capture device with image correction and extracts a tongue color feature vector from each image. This vector consists of 12 color ratios calculated with the tongue color gamut to better characterize each foreground tongue pixel. Testing was carried out on a large dataset collected from Guangdong, China, consisting of 143 Healthy and 902 Disease images 
TABLE 6: Mean tongue color features of the three clusters.

\begin{tabular}{lcccccrr}
\hline Cluster number & R & DR & LR & BK & GY & W & Y \\
\hline 1 & 21.561 & 13.972 & 12.265 & 12.535 & 9.524 & 3.703 & 26.191 \\
2 & 17.116 & 11.980 & 9.437 & 15.574 & 34.733 & 4.111 & 6.539 \\
3 & 40.736 & 15.396 & 15.872 & 8.232 & 10.770 & 1.668 & 7.015 \\
\hline
\end{tabular}

TABLE 7: Classification result between the three clusters compared in groups of two.

\begin{tabular}{lc}
\hline Cluster comparison & Average accuracy \\
\hline Cluster 1 versus Cluster 2 & $100 \%$ \\
Cluster 1 versus Cluster 3 & $97.75 \%$ \\
Cluster 2 versus Cluster 3 & $99.63 \%$ \\
\hline
\end{tabular}

TABLE 8: Classification result of the illnesses.

\begin{tabular}{lccc}
\hline Disease ID & Cluster number & $k$-NN & SVM \\
\hline 1 & 1 & $72.53 \%$ & $76.08 \%$ \\
2 & 3 & $54.87 \%$ & $54.41 \%$ \\
3 & 3 & $72.97 \%$ & $75.68 \%$ \\
4 & 3 & $54.87 \%$ & $54.41 \%$ \\
5 & 2 & $81.45 \%$ & $81.45 \%$ \\
6 & 3 & $78.61 \%$ & $74.05 \%$ \\
7 & 2 & $83.33 \%$ & $93.06 \%$ \\
8 & 2 & $72.02 \%$ & $83.33 \%$ \\
9 & 2 & $77.78 \%$ & $83.33 \%$ \\
10 & 2 & $78.71 \%$ & $87.10 \%$ \\
11 & 3 & $72.82 \%$ & $73.56 \%$ \\
12 & 2 & $81.45 \%$ & $81.45 \%$ \\
13 & 1 & $72.53 \%$ & $76.08 \%$ \\
\hline
\end{tabular}

(13 specific illnesses with at least 10 samples and a miscellaneous folder). The experimental results showed that there is a relationship between tongue color and the state of the human body, which can be used in medical applications to detect various illnesses. This can potentially lead to a new painless and efficient way to examine patients.

\section{Acknowledgments}

The work is partially supported by the GRF fund from the HKSAR Government, the central fund from Hong Kong Polytechnic University, and the NSFC Oversea fund (61020106004), China.

\section{References}

[1] C. C. Chiu, "Development of a computerized tongue diagnosis system," Biomedical Engineering, vol. 8, no. 4, pp. 342-350, 1996.

[2] C. C. Chiu, "A novel approach based on computerized image analysis for traditional Chinese medical diagnosis of the tongue," Computer Methods and Programs in Biomedicine, vol. 61, no. 2, pp. 77-89, 2000.

[3] R. O. Duda, P. E. Hart, and D. G. Stork, Pattern Classification, Wiley \& Sons, 2nd edition, 2000.
[4] B. Kirschbaum, Atlas of Chinese Tongue Diagnosis, Eastland Press, Seattle, Wash, USA, 2000.

[5] B. Li, Q. Huang, Y. Lu, S. Chen, R. Liang, and Z. Wang, "A method of classifying tongue colors for traditional chinese medicine diagnosis based on the CIELAB color space," in Proceedings of the International Conference on Medical Biometrics, pp. 153-159, 2007.

[6] C. H. Li and P. C. Yuen, "Tongue image matching using color content," Pattern Recognition, vol. 35, no. 2, pp. 407-419, 2002.

[7] N. M. Li, "The contemporary investigations of computerized tongue diagnosis," in The Handbook of Chinese Tongue Diagnosis, Shed-Yuan Publishing, 1994.

[8] G. Maciocia, Tongue Diagnosis in Chinese Medicine, Eastland Press, Seattle, Wash, USA, 1995.

[9] N. Ohta and A. Robertson, Colorimetry: Fundamentals and Applications, John Wiley \& Sons, 2006.

[10] B. Pang, D. Zhang, and K. Wang, "Tongue image analysis for appendicitis diagnosis," Information Sciences, vol. 175, no. 3, pp. 160-176, 2005.

[11] B. Pang, D. Zhang, and K. Wang, "The bi-elliptical deformable contour and its application to automated tongue segmentation in chinese medicine," IEEE Transactions on Medical Imaging, vol. 24, no. 8, pp. 946-956, 2005.

[12] W. Su, Z. Y. Xu, Z. Q. Wang, and J. T. Xu, “Objectified study on tongue images of patients with lung cancer of different syndromes," Chinese Journal of Integrative Medicine, vol. 17, no. 4, pp. 272-276, 2011.

[13] K. Q. Wang, D. Zhang, N. M. Li, and B. Pang, "Tongue diagnosis based on biometric pattern recognition technology," in Pattern Recognition from Classical to Modern Approaches, S. K. Pal and A. Pal, Eds., pp. 575-598, World Scientific, 1st edition, 2001.

[14] X. Wang and D. Zhang, "An optimized tongue image color correction scheme," IEEE Transactions on Information Technology in Biomedicine, vol. 14, no. 6, pp. 1355-1364, 2010.

[15] X. Wang and D. Zhang, "Statistical tongue color distribution and its application," in Prococeedings of the International Conference on Computer and Computational Intelligence, 2011.

[16] D. Zhang, Automated Biometrics: Technologies and Systems, Kluwer Academic Publisher, Boston, Mass, USA, 2000.

[17] D. Zhang, B. Pang, N. Li, K. Wang, and H. Zhang, "Computerized diagnosis from tongue appearance using quantitative feature classification," The American Journal of Chinese Medicine, vol. 33, no. 6, pp. 859-866, 2005.

[18] H. Z. Zhang, K. Q. Wang, X. S. Jin, and D. Zhang, "SVR based color calibration for tongue image," in Proceedings of the International Conference on Machine Learning and Cybernetics (ICMLC '05), pp. 5065-5070, August 2005.

[19] Y. Zhang, R. Liang, Z. Wang, Y. Fan, and F. Li, "Analysis of the color characteristics of tongue digital images from 884 physical examination cases," Journal of Beijing University of Traditional Chinese Medicine, vol. 28, pp. 73-75, 2005. 


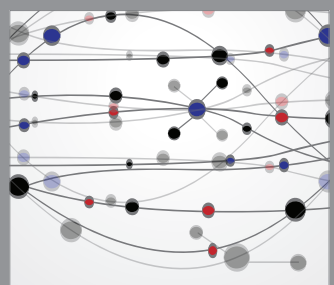

The Scientific World Journal
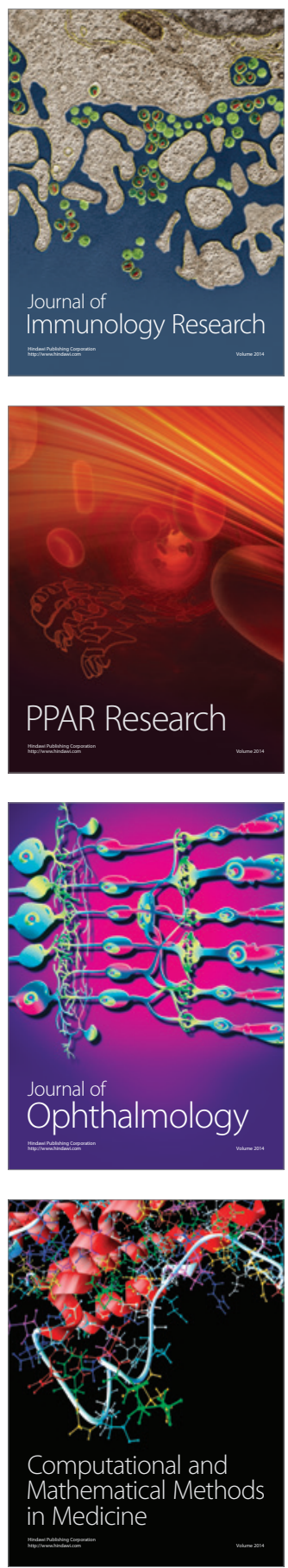

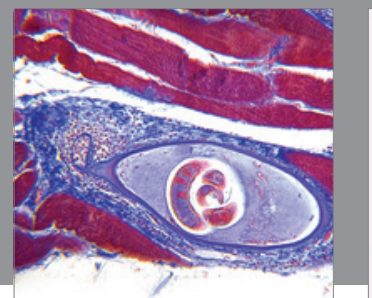

Gastroenterology

Research and Practice
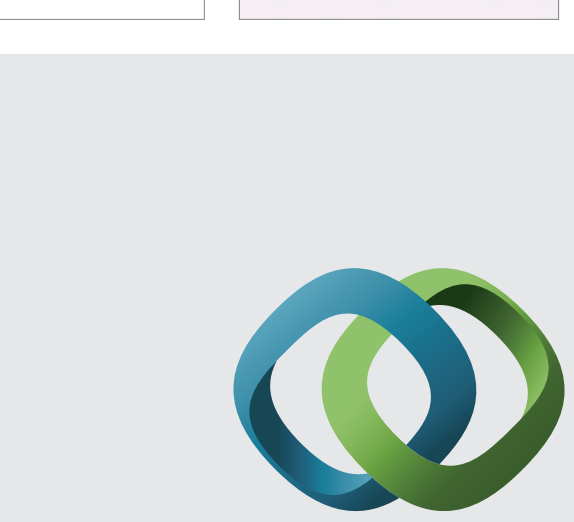

\section{Hindawi}

Submit your manuscripts at

http://www.hindawi.com
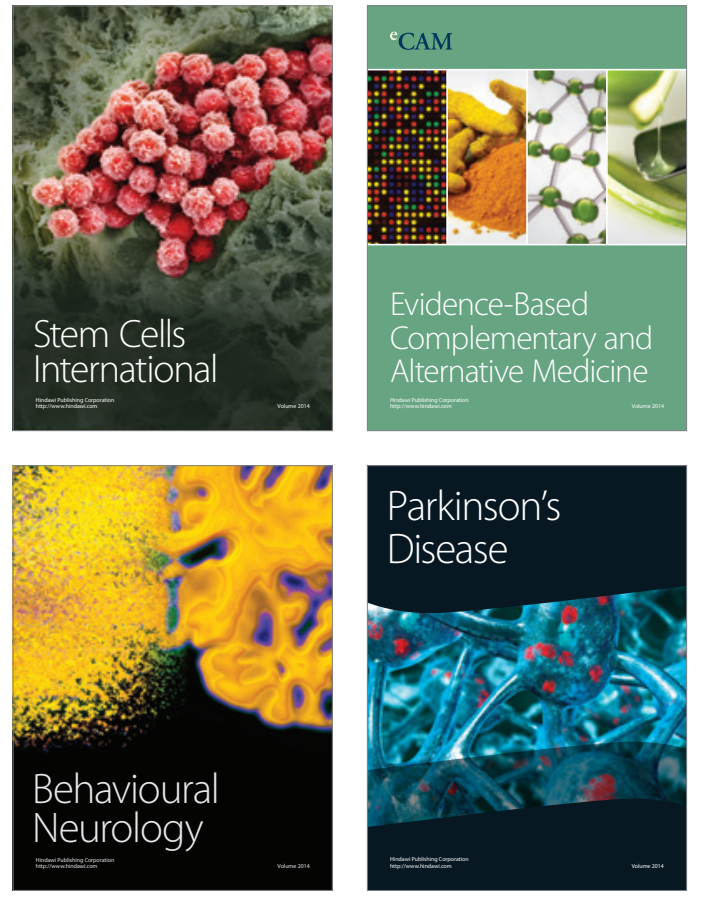
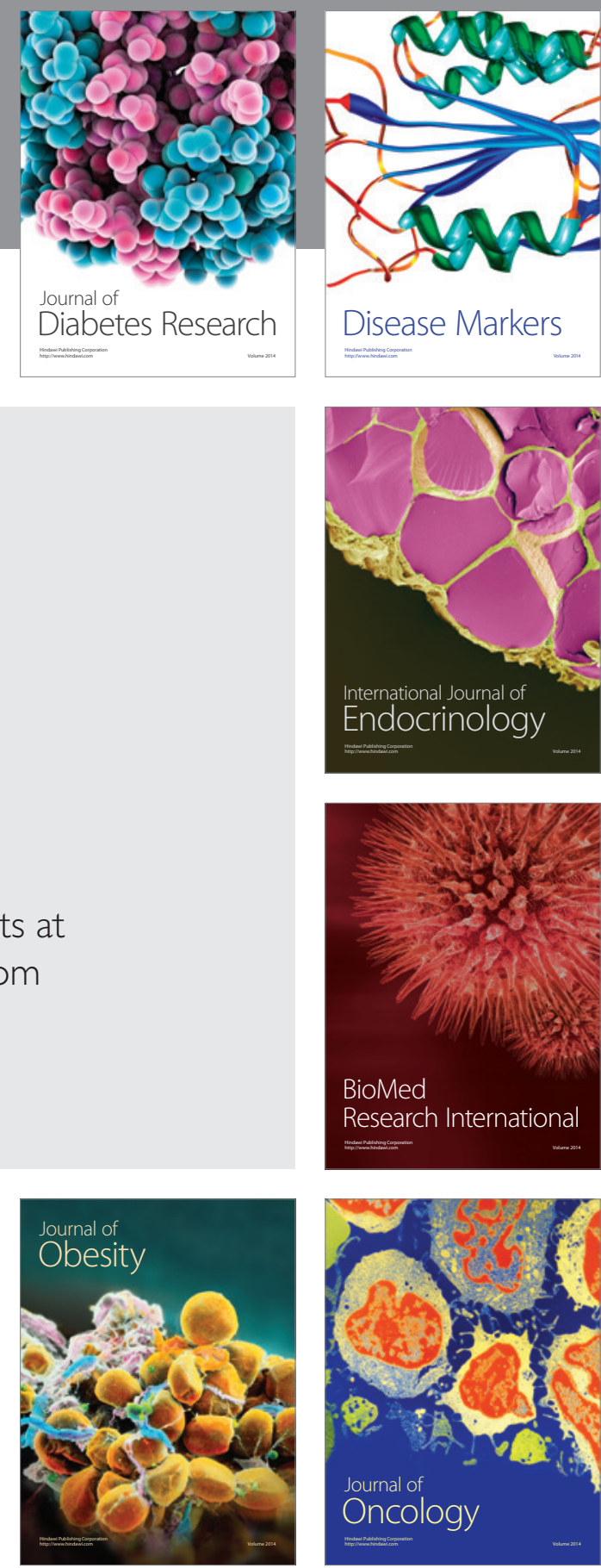

Disease Markers
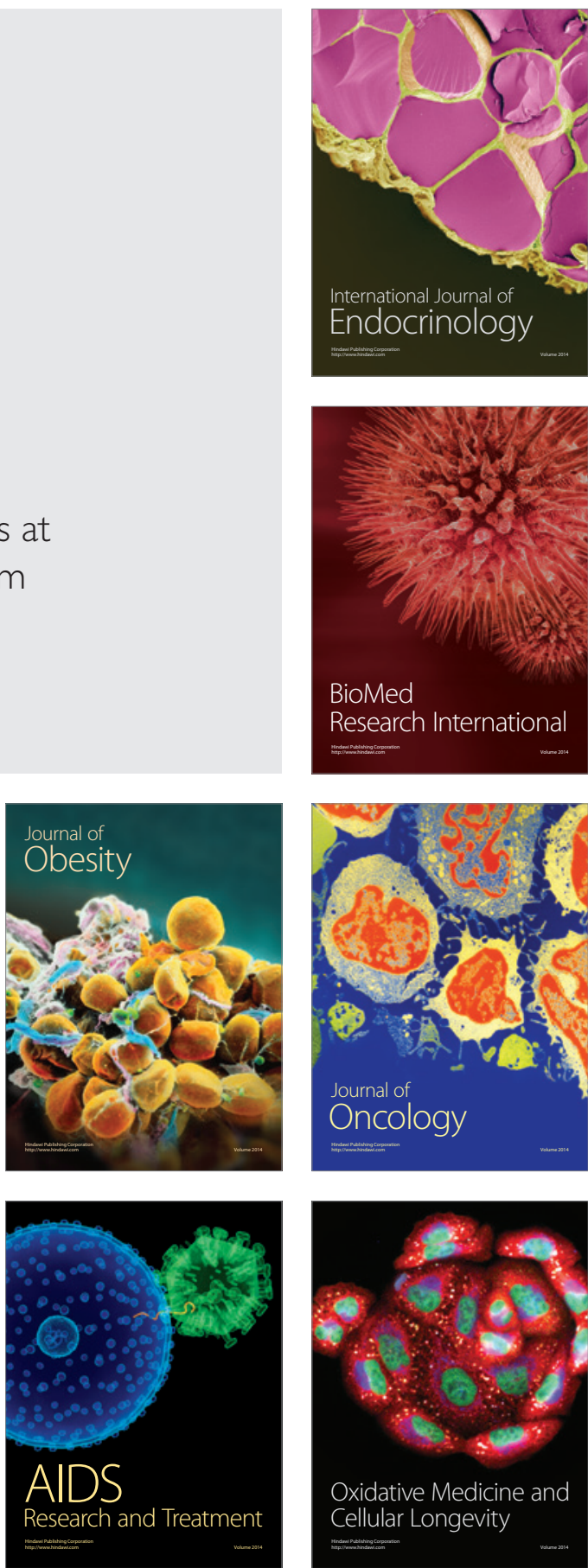